\title{
Upconverting nanorockers for intracellular viscosity measurements during chemotherapy
}

Paloma Rodríguez-Sevilla*, Francisco Sanz-Rodríguez, Raúl P. Peláez, Rafael DelgadoBuscalioni, Liangliang Liang, Xiaogang Liu, and Daniel Jaque

Dr P. Rodríguez-Sevilla

SUPA, School of Physics and Astronomy, University of St Andrews, North Haugh, Fife, KY16 9SS, United Kingdom

Dr F. Sanz-Rodríguez

Fluorescence Imaging Group, Departamento de Biología, Facultad de Ciencias, Universidad Autónoma de Madrid, 28049 Madrid, Spain.

R. P. Peláez, Prof R. Delgado-Buscalioni

Departamento de Física Teórica de la Materia Condensada, Facultad de Ciencias, Universidad Autónoma de Madrid, 28049 Madrid, Spain.

Liangliang Liang, Prof Xiaogang Liu

Department of Chemistry, National University of Singapore, Science Drive 3, Singapore 117543, Singapore.

Dr Daniel Jaque

Fluorescence Imaging Group, Departamento de Fisica de Materiales, Universidad Autónoma de Madrid, 28049 Madrid, Spain

Nanobiology Group, Instituto Ramón y Cajal de Investigación Sanitaria Hospital Ramón y

Cajal. Ctra. De Colmenar Viejo Km. 9100, 28034 Madrid, Spain

E-mail:prs8@st-andrews.ac.uk

Keywords: Upconverting nanoparticles, in vitro chemotherapy, rheometry, cancer

Chemicals capable of producing structural and chemical changes on cells are used to treat diseases (e.g. cancer). Further development and optimization of chemotherapies require thorough knowledge of the effect of the chemical on the cellular structure and dynamics. This involves studying, in a non-invasive way, the properties of individual cells after drug administration. Intracellular viscosity is affected by chemical treatments and it can be reliably used to monitor chemotherapies at the cellular level. Here, cancer cell monitoring during chemotherapeutic treatments is demonstrated using intracellular allocated upconverting nanorockers. A simple analysis of the polarized visible emission of a single particle provides 


\section{WILEY-VCH}

a real-time readout of its rocking dynamics that are directly correlated to the cytoplasmic viscosity. Numerical simulations and immunodetection are used to correlate the measured intracellular viscosity alterations to the changes produced in the cytoskeleton of cancer cells by anticancer drugs (colchicine and Taxol). This study evidences the possibility of monitoring cellular properties under an external chemical stimulus for the study and development of new treatments. Moreover, it provides the biomedical community with new tools to study intracellular dynamics and cell functioning. 


\section{WILEY-VCH}

\section{Introduction}

Rheological parameters, such as creep compliance, viscosity or elasticity, are of great importance at the cellular level. Cytoplasmic viscosity controls the diffusion and transport of molecules and organelles inside the cell. It is also a reliable indicator of the cellular state, which can be used to detect the appearance and onset of different diseases, including cancer. ${ }^{[1]}$ The precise knowledge of the intracellular viscosity can be used to evaluate the effects caused by different treatments at the single-cell level. Indeed, cytoplasmic viscosity is modified when cancer cells are subjected to a chemotherapy process, so that variations in the intracellular viscosity can be used to assess the evolution of the treatment. ${ }^{[2]}$ The intracellular environment is a composite of water and a variety of proteins and organelles. One of the main protein components of the cytoplasm is the cytoskeleton. This three-dimensional network composed of filamentous proteins is a highly mobile, viscoelastic and flexible entity that controls the cellular mechanics. ${ }^{[3]}$ Particularly, alterations produced in the intracellular viscosity due to drug administration are governed by the effect of the chemical on the cytoskeleton of the cell. There are three major types of cytoskeletal filaments: microtubules, actin filaments, and intermediate filaments. They differ from each other in their molecular structure, function and mechanical properties. These filamentous proteins, and other associated proteins, have very different dynamics and changes in their local concentration affect the viscosity of the cytoplasm.

After many years of research, various natural, semi-synthetic and synthetic compounds have been developed to control the growth of cancer cells or tissue. These anticancer agents are broadly classified as alkylating agents, antimetabolites, antibiotics, natural products (e.g. colchicine and Taxol), and hormones. ${ }^{[4]}$ Anticancer drugs mainly prevent cell division by blocking sites of action and inhibiting enzyme release. Based on their targets, anti-cancer agents are classified into tubulin and non-tubulin interactors. Tubulin is a heterodimer formed 


\section{WILEY-VCH}

by two globular protein subunits, $\alpha$ - and $\beta$-tubulin. ${ }^{[5]}$ They polymerize to form the microtubules, which are in a continuous dynamic cycle of polymerization and depolymerization. Through these dynamic mechanisms, microtubules are one of the important components of cell division: they produce the mitotic spindle at anaphase to form new daughter cells. ${ }^{[6]}$ Drugs that interfere with the polymerization and depolymerization of microtubules are called tubulin interactors. ${ }^{[7]}$ Depending on their interference action, they are classified into two categories. ${ }^{[8]}$ Tubulin destabilizing agents, such as colchicine, inhibit tubulin polymerization, causing the shortening of the microtubules and a net decrease in its number. ${ }^{[9]}$ On the other hand, tubulin stabilizing agents, such as Taxol, stabilize microtubules, enhance tubulin polymerization, and reduce tubulin heterodimers concentration in the cytoplasm. ${ }^{[5 \mathrm{a}]}$ In either case the microtubule dynamics are disrupted and the mitotic spindle cannot be formed. ${ }^{[10]}$

Unfortunately, these drugs are associated with severe side effects in somatic cells and tissues due to the non-selectivity of the anti-cancer agents toward the cancer cells. Specificity in the treatment is an important aspect in order to reduce the toxicity, whereas it is difficult to obtain because cancer cells are similar to normal cells. ${ }^{[11]}$ However, the treatment will affect differently each type of cell due to their different division rate. Most somatic cells in the body are not dividing, so drugs actin on microtubule dynamics have little effect on their cell division. On the other hand, tumor cells have a high rate of proliferation which makes them more likely to be affected by the treatment, favoring the inhibition of tumor growth. Each type of tubulin interactor has a different impact on the intracellular viscosity so that the monitoring of the viscosity changes can be used to control the efficacy of the treatment at the single-cell level. Such monitoring must be done in a non-invasive (contactless) way so that the measured changes could be only attributed to the effect of the administrated drug. Measuring cytoplasm properties in such an invasive way is a challenging endeavor. Through 


\section{WILEY-VCH}

decades, different rheometric techniques have been developed for single-cell studies. ${ }^{\text {[1a, } 12]}$ They can be divided into passive and active methods. Active techniques impose an external force in order to produce a controlled deformation of the cell to measure its reaction to the applied stimulus. ${ }^{[13]}$ On the other hand, passive techniques are based on the analysis of the thermal fluctuations of endogenous (intracellular material) and exogenous (particles) entities inside the cell. ${ }^{[1 \mathrm{a}, 2 \mathrm{c}, 14]}$ These technics are less invasive than active methods and can be combined with luminescent nanoparticles or molecules to improve spatial resolution. ${ }^{[15]}$ Single-particle spectroscopy has served to reduce the number of agents (ideally just one) required for imaging or sensing. This technique can be combined with passive rheometric techniques for viscosity measurements. For this purpose, upconverting particles (UCPs) are the perfect candidate ought to their outstanding luminescent properties and biocompatibility. This kind of luminescent particles can produce visible emission under infrared excitation via a multiphoton excitation process. ${ }^{[16]}$ Moreover, stable and non-blinking emission enables prolonged measurements of the luminescence of a single UCP, required for specific studies. In addition, the shape and size of UCPs can be tailor-made for the phenomenon to be studied. As an example, the polarized emission of non-spherical UCPs gives additional information on the dynamics of the particle (i.e. not only location and translation, but also rotation) inside a liquid and it can serve to extract information of the viscosity of the surrounding medium. ${ }^{[17]}$ Moreover, UCPs of sizes ranging from few nanometers to tens of micros can be used to explore viscosity in a wide range of scales. As it will be explained later, the measured value of the intracellular viscosity strongly depends on the size of the agent used to measure it. ${ }^{[18]}$ A non-spherical particle (i.e. disk) inside a fluid, in addition to diffuse, "rocks" due to the collision of the molecules of the fluid with the particle. The magnitude of this angular vibration depends not only on temperature, but also on the viscosity of the fluid. This vibrational movement can be characterized by the mean square angular displacement 


\section{WILEY-VCH}

(MSAD), whose evolution with time $(t)$, in the particular case of a disk-shaped particle, is given by

$\operatorname{MSAD}(t)=\frac{k_{B} T}{3 \eta V f / f_{0}} t$.

This equation takes into account the intracellular viscosity $(\eta)$, the thermal energy $\left(k_{B} T\right.$, where $k_{B}$ is the Boltzmann constant, and $T$ the temperature) and the characteristics of the particle (volume, $V$, and Perrin's friction coefficient, $f / f_{0}$, a parameter that relates the friction of the non-spherical object with that of a sphere of equivalent volume). ${ }^{[19]}$ Thus, the time evolution of the MSAD of a non-spherical particle inside a cell provides a direct measurement of the cytoplasmic viscosity.

Determining the time evolution of the MSAD of a nanoparticle inside a living cell is challenging. Conventional approaches, such as video-based techniques cannot be applied due to the small dimensions of the particle. ${ }^{[14 \mathrm{~d}, 20]}$ In this work, we face this problem by using non-spherical upconversting nanoparticles (UCNPs). Our nanorockers are disk-shaped $\beta$ $\mathrm{NaYF}_{4}$ nanoparticles with a mean radius and thickness of $400 \mathrm{~nm}$, as shown in the SEM image of Figure 1a. This particle's size allows us to measure changes in the mesoscopic viscosity of the cell. As mentioned above and will be discussed later, the viscosity that affects the movement of the particle depends on its relative size to that of the intracellular components. Very small particles are only able to measure the cytosolic viscosity, whose value is of the order of that of water. External agents (i.e. drugs) do not affect the cytosolic but the mesoscopic viscosity. For this reason, we select this particle size. The nanoparticles are doped with $\mathrm{Er}^{3+}$ and $\mathrm{Yb}^{3+}$ ions. This combination of dopants and host material ensured an intense visible emission (500-700 nm) under $980 \mathrm{~nm}$ excitation. Due to the particular crystal structure of $\beta-\mathrm{NaYF}_{4}$, our disk-shaped nanorockers showed a red emission band that is highly polarized. Therefore, for a fixed detection geometry, the spectral shape of the emission depends on the angular position of the nanorocker. This makes possible a straightforward 


\section{WILEY-VCH}

measurement of the time evolution of the MSAD. This, in turn, allows us to determine the evolution of intracellular viscosity during cancer cell treatment by a simple analysis of the fluctuations in the luminescence of the disk-shaped UCNP produced by its vibration. In the present study, we have used upconverting nanorockers to monitor single-cell chemotherapeutic processes based on two different anticancer drugs, colchicine and Taxol, using the intracellular viscosity as a diagnostic parameter. We correlated the effect of these chemicals in the intracellular viscosity to the structural changes of the treated cells. We also developed a theoretical model to understand the role played by the cytoskeleton of the cell in the viscosity changes.

\section{Experimental design}

Our experimental setup is depicted in Figure 1a and described in the experimental section. Briefly, a $980 \mathrm{~nm}$ laser beam is focused using a microscope objective to excite the luminescence of the target UCNP. We use the same objective to collect the luminescence and to image the sample plane using a CMOS camera. The luminescence spectra are analyzed by using a CCD camera coupled to a monochromator.

We used HeLa cancer cells (ATCC) to test the performance of the chemotherapeutic treatments (see the experimental section for details). The UCNPs are incorporated by the cells prior to the chemical treatment. Once inside the cell, the rocking dynamics of the UCNP are influenced by its interaction with the intracellular components, such as microtubules (schematically represented in Figure 1b, c and d) and the local density of tubulin heterodimers (Figure 1d).

The rheometric technique we used encompasses four main steps: (i) measurement of the polarized luminescence emission of the internalized UCNP, (ii) analysis of these spectra to obtain the angular displacement of the particle with time, (iii) determination of the MSAD 
from the angular displacement, and (iv) fitting of the MSAD to Equation 1 to obtain a value of the intracellular viscosity (see the experimental section for more details).

We determined the temporal evolution of the MSAD of the upconverting nanorockers by analyzing the spectral fluctuations of their polarized visible emission. ${ }^{[21]}$ The intensity ratio $\left(R=I_{654} / I_{661}\right)$ of the peaks centred at $654 \mathrm{~nm}$ and $661 \mathrm{~nm}$ (highlighted in Figure 2a) strongly depends on the orientation of the particle. Thus, the angular position $(\theta)$ can be elucidated from the relative emission at 654 and $661 \mathrm{~nm}$ using

$\theta(R)=\operatorname{acos}\left(\sqrt{\frac{R_{\max }-R}{R_{\max }-R_{\min }}}\right)$,

where $R_{\min }=R(\theta=0)$ is the minimum value of the intensity ratio reached when the particle is horizontally orientated (i.e. with the largest facet perpendicular to the beam propagation direction). Similarly, $R_{\max }=R(\theta=\pi / 2)$ is the maximum value when the particle is vertically orientated (i.e. its largest facet parallel to the beam propagation direction). To determine the angular thermal fluctuations of the internalized nanorocket (i.e. the $\theta$ vs $t$ curve), we recorded consecutive luminescent spectra for a total measurement time of approximately 4 minutes (set of gray spectra in Figure 2a). The used laser intensity was approximately $10^{6} \mathrm{~W} / \mathrm{cm}^{2}$, which ensures a good signal-to-noise ratio without producing cell damage. The UCNP must be in the vertical orientation to reduce the action of optical torques that can interfere in the Brownian dynamics of the particle (see supporting information). ${ }^{[17]}$ Then, we used the measured luminescent spectra to compute the intensity ratio $R$ (see Figure $2 b$ ), which was utilized to obtain a value of the angular displacement of the particle using Equation 2. It is worth noting that the determination of the orientation of the particle is made by a ratiometric technique. This ensures that, as long as the signal-to-noise ratio is high enough to give a resolved spectrum, the value of the intensity ratio does not depend on the total emission intensity. Finally, we calculated the MSAD. Figure 2c shows, as an illustrative example, the averaged MSAD measured as a function of time for cells incubated for 0.5 


\section{WILEY-VCH}

(black) and 5 (blue) hours with Taxol at $0.2 \mu \mathrm{M}$ concentration. The linear fit of these curves to Equation 1 gives the mean value of the intracellular viscosity of the cells subjected to the drug treatment. Note the change in the slope of the MSAD vs time curves that evidences a change in the intracellular viscosity as a consequence of Taxol administration, as it will be discussed in detail later.

\section{Results and discussion}

\subsection{Intracellular viscosity changes}

We used the above-described rheometric technique to quantify the intracellular viscosity of HeLa cells when subjected to different chemotherapeutic treatments (see experimental section for details). We treated the cells for different periods of time with $0.2 \mu \mathrm{M}$ of Taxol, and with 0.1 , and $1 \mu \mathrm{M}$ colchicine during $2 \mathrm{~h}$. We selected these drug concentrations and treatment times because previous studies had shown that they are non-toxic to cells while able to produce changes in the intracellular viscosity. Hence, we expected to see similar effects on HeLa cells. Results are shown in Figure 3. Data correspond to the mean value of all measurements for the given treatment conditions and error bars represent the standard deviation. The main source of uncertainty in our measurements is the variability in the cell population. For that reason, we chose this parameter as our experimental error. Error propagation in Equations 1 and 2 give a smaller uncertainty in the viscosity measurement $(\sim 2$ $\%)$.

In the case of Taxol (Figure 3a), the intracellular viscosity decreases with the duration of the treatment. An exponentially decreasing function was fitted to the experimental data, which gave a decay ratio of 0.8 hours, similar to that published for macrophages $(1.16 \mathrm{~h})$ for a drug concentration of $0.2 \mu \mathrm{M} .{ }^{[2 \mathrm{c}]}$ In addition, results obtained on Taxol-treated neutrophils reveal a negligible change in the intracellular viscosity for an incubation time of around $20 \mathrm{~min}$ at a concentration five times larger than the used in this work. ${ }^{[2 b]}$ Even though the effect of the 


\section{WILEY-VCH}

drugs is not expected to be the same on different cell types due to their structural and functional dissimilarities, our results and those published in the literature suggest that the action of Taxol on the intracellular viscosity, at a particular concentration, is time-dependent. On the other hand, the treatment with colchicine produces the reverse effect: as the concentration of the drug increases, the viscosity also does (Figure 3b). These results additionally suggest that there is a threshold (in our case larger than $0.1 \mu \mathrm{M}$ ) above which the viscosity is affected by the drug. Our results show that $0.1 \mu \mathrm{M}$ of colchicine has a negligible effect on cell viscosity since the measured viscosity is, within the experimental error, the same as that obtained for the untreated cells. This concentration-dependent effect and threshold were also observed for colchicine-treated neutrophils. ${ }^{[2 b]}$ In this published work, colchicine concentration had to be increased up to $10 \mu \mathrm{M}$ in order to see a clear increase in the intracellular viscosity. Moreover, the two-fold increase in the viscosity showed in Figure $3 \mathrm{~b}$ was achieved for that type of cells at a concentration as high as $100 \mu \mathrm{M}$.

It is worth noting that due to their size, the used UCNPs are internalized via endocytosis. This could lead to the formation of a membrane between the particle and the cytoplasm. In that case, one would expect that the presence of the membrane would affect the measurement regardless of the treatment, thus giving the same viscosity value irrespectively of the cell state. However, our results show that there is a change in the intracellular viscosity after drug administration that affects the particle's movement. We conclude that this membrane, if present, does not prevent the measurement of the viscosity changes, but it may affect the absolute value of the measured viscosity.

\subsection{Structural changes}

Once the effect of Taxol and colchicine on the intracellular viscosity was determined, we assessed the structural changes induced on the cytoskeleton of HeLa cells by these drugs through immunodetection (see the experimental section for details). We show the distinct 


\section{WILEY-VCH}

action of Taxol and colchicine on microtubule structure in the upper panels of Figure 4 and Figure S2 of the supporting information. We show the expected microtubule stabilization produced by Taxol in the second and third upper panels of Figure 4. The main difference between control interphase HeLa cells (first upper panel) and Taxol-treated cells is the formation of microtubule bundles. Control cells present an irregular structure of microtubules, while in Taxol-treated cells the density of microtubules increases and they orient parallelly forming bundles. This is in good agreement with results obtained in HeLa cells treated with a micromolar concentration of Taxol. ${ }^{[22]}$ We cannot distinguish any mayor difference between the tubulin network of cells incubated for 2 and $5 \mathrm{~h}$. This is in good agreement with the similarity in the viscosity values measured for those treatment times (see Figure 3a). However, we found differences between control and $5 \mathrm{~h}$ incubation time (see large field image in Figure S2) in the morphology of Hela cells. As incubation time increases, treated cells tend to present a more rounded shape than control cells. In addition, we observed that the number of cells in a state between metaphase and anaphase in the cycle of cell division (mitosis) increases. This indicates that cells affected by Taxol during mitosis cannot longer complete the process since microtubules have lost their dynamic behavior. ${ }^{[22]}$

On the other hand, we observed that colchicine presents the opposite effect on microtubules to Taxol, as shown by previous studies. ${ }^{[23]}$ In this case, microtubules cannot longer be formed thus they remain in the cytoplasm as dimers, as we show in the fifth upper panel of Figure 4. When HeLa cells were incubated with $1 \mu \mathrm{M}$ of Colchicine, microtubules lost their structure in the form of filaments and consequently the microtubule network was disrupted. We did not see this effect in the cells treated with $0.1 \mu \mathrm{M}$ of colchicine (fourth upper panel) which is in good agreement with the viscosity measurements shown in Figure $3 \mathrm{~b}$. 


\section{WILEY-VCH}

In the case of the actin cytoskeleton, we did not observe changes in the distribution of F-actin in the cell cytoplasm for any of the treatments, as shown in the bottom panels of Figure 4. Actin filaments remained distributed in the form of stress fibers and cortical fibers, as in the case of control cells. ${ }^{[24]}$ These results suggest that the viscosity changes we have measured are primarily due to alterations in the microtubule cytoskeleton. This is in good agreement with previous studies on the HeLa cells treated with Taxol and colchicine. ${ }^{[23]}$

\subsection{Simulations}

Once structural changes in the treated cells have been discussed, these need to be related to the changes in the intracellular viscosity. The immunodetection assay suggests that modifications in the microtubule structures are the origin of the viscosity changes. Before drug administration, the cytoplasm activity includes the interaction of tubulin dimers with different types of microtubule-associated proteins, which form aggregates. ${ }^{[25]}$ It is the interaction of these dynamic aggregates, rather than a lateral assembly of individual dimers, which produces the formation of protofilaments and short microtubule strands. In this scenario, the cytoplasm behaves as a transient gel with aggregated structures. Notably, the resulting mesoscopic structures have an average size similar to that of the used nanorokers (400 nm). This fact is important because the viscosity of complex fluids, such as the cytoplasm, depends on the length-scale of the sample since the Brownian motion of a particle in a disordered media samples the local viscosity of length-scales comparable to its size. ${ }^{[18]}$ Therefore, the particle will be affected by an effective viscosity which value depends on its size in respect to the characteristic size of the fluid components. If the particle is smaller than the typical size of the aggregates $\left(R_{p}<<R_{a}\right.$, being $R_{p}$ and $R_{a}$ the particle and aggregate effective radius, respectively), it samples the viscosity of the solvent where the aggregates are dispersed (i.e. cytosolic viscosity). In the mesoscopic regime, when the particle is comparable 
to the aggregates, the effective viscosity increases exponentially with $R_{p} / R_{a}$ until it saturates to a macroscopic value for $R_{p}>>R_{a \cdot}{ }^{[18]}$

For a typical concentration of tubulin $(25-100 \mu \mathrm{M})$ in the cytoplasm, the average volume fraction of microtubules is between $5 \times 10^{-3}$ and $10^{-2}$. At these concentrations, a polydisperse ensemble of rods (i.e. microtubules) with attractive interactions form a fractal gel whose pores are typically smaller than the average length of the rods. ${ }^{[26]}$ Accurate simulations of the diffusion of the nanorocker in such transient network of short filaments go beyond the present contribution as one would require knowledge of, among other factors, the polydispersity of the filaments and the interaction potentials. Nevertheless, the diffusion of tracer particles in gels present a generic behavior and, in particular, owing to the steric hindrance of the gel obstacles, both translational and rotational diffusions are severely reduced typically by one order of magnitude and often leading to anomalous diffusion. ${ }^{[26-27]}$

Such scenario gradually changes upon administration of Taxol. This drug induces an irreversible polymerization of microtubules over time, leading to an increase of the average size of the gel pores which favors the rotational diffusion of the nanorocker. The 10 -fold viscosity decrease observed in Figure $3 \mathrm{a}$ indicates that, $5 \mathrm{~h}$ after the administration of Taxol, the particle is moving around significantly larger structures, i.e. microtubules. Therefore, it is sampling scales much shorter than the microtubules and consequently detects a smaller effective viscosity. On the other hand, colchicine prevents the formation of filaments and aggregates, reducing the average length of the cytoplasm components that drag the particle. In this situation, the particle is affected by a larger effective viscosity.

In addition, in a suspension of rods with a volume fraction of $\sim 0.01$, a transition from disordered to ordered phase takes place once the rods become larger than about $2 \mu \mathrm{m} .{ }^{[26]}$ This length scale is not far from the nanoroker diameter used here (i.e. $800 \mathrm{~nm}$ ). In the ordered 


\section{WILEY-VCH}

phase, the typical distance between microtubules becomes larger than the nanoroker size and the steric hindrance of the microtubules is significantly reduced. Figure $3 \mathrm{a}$ indicates that the transition to the ordered phase could take place after $2 \mathrm{~h}$ of Taxol administration.

To validate this hypothesis, we studied whether or not the mobility of the particle is significantly altered by the presence of long microtubules resulting from a prolonged $(5 \mathrm{~h})$ incubation with Taxol. We performed numerical simulations based on the immersed boundary method for Brownian hydrodynamics, which reproduce the particle environment after the administration of Taxol. ${ }^{[28]}$ Simulations were carried out in periodic boxes of side $L$ $=64 \times h$, where $h$ is the unit-mesh size, whose value was chosen based on the particle size. As shown in Figure 5a, the particle is built up by using a number of spherical beads (of radius $\mathrm{R}_{\mathrm{h}} \approx h$ ) connected by a network of hard springs to ensure a negligible deformation. The width of the particle (400 $\mathrm{nm}$ ) was set to 8 beads, which approximately corresponded to a particle's hydrodynamic radius of $14 \times h$, so that $h \approx 28.6 \mathrm{~nm}$ and $L \approx 1.83 \mu \mathrm{m}$.

Microtubules were similarly constructed using 3 strands of beads connected with three-body potentials, providing a large bending rigidity (see Figure 5a).

Using a reference concentration of tubulin of $25 \mu \mathrm{M}$ and a microtubule diameter of approximately $50 \mathrm{~nm},{ }^{[25]}$ we estimate that the average number of microtubules crossing a surface of one micron square is about 2, although this number varies over the cell. We performed simulations with an increasing number of microtubules, with a maximum value of 20, which corresponds to 6 microtubules per micron square. Two different scenarios were considered taking into account the presence or not of the excitation beam. In the "free" case, the particle is free to translate and collide with the microtubules. In the "confined" case, the particle feels an external potential which confines its translation (but not its rotation) to a domain which is about $10 \%$ larger than its volume. In this confined case, any modification of the particle rotation is only due to effects of the microtubules on the hydrodynamic mobility 


\section{WILEY-VCH}

of the particle. We note that, in the experiments, the particle might be softly confined due to the optical forces. Therefore, a comparison between both scenarios enables a cross-check of the significance of the viscosity measurement. We calculated the MSAD for both scenarios and, additionally, the translational diffusion (i.e. mean square displacement, MSD) for the "free" case. The theoretical MSAD of a perfect disk with the same aspect ratio as our nanorokers, given by Equation 1, is plotted in Figure 5b (orange line). This curve matches, within statistical uncertainty, to the MSAD obtained with simulations in the absence of microtubules, validating the approximation of the UCNP to a disk. We then added microtubules to simulate the modification of the viscosity in the "confined" case. Figure 5b shows that, up to the concentrations considered, the viscosity sensed by the particle is practically not modified by the presence of microtubules. We can conclude from this that, once all the microtubules are formed, there is a less amount of intracellular material of comparable size to the nanorocker, therefore the Brownian motion of the particle is affected by a reduced viscosity. Finally, the simulated MSAD for the free and confined cases are similar, showing that the translational confinement of the particle by the excitation beam does not restrict or affect its rocking motion.

\section{Conclusion}

In summary, we use the intracellular rocking dynamics of disk-shaped upconverting nanoparticles for the determination of the intracellular viscosity of HeLa cancer cells subjected to anticancer drugs (i.e. Taxol and colchicine). A simple analysis of the time evolution of the visible emission generated by the internalized nanorockers allowed us to determine the time evolution of their accumulated angular displacement and, from this, the intracellular viscosity. Our results show a correlation between drug administration and alterations in the intracellular viscosity of HeLa cells, so that accurate monitoring of the treatment becomes possible. We have observed that the intracellular viscosity of HeLa cells 


\section{WILEY-VCH}

decreases as the incubation time with Taxol increases. On the other hand, as the concentration of colchicine is increased above a threshold, the viscosity of the cell also does. These results are comparable to those obtained for neutrophil and macrophage cells treated with the same drugs.

In addition, we analyze the experimentally measured viscosity in terms of the structural changes produced on the cytoskeleton of the cells and numerical simulations of the diffusion of the nanorocker, to understand the effect of the studied chemical treatments on HeLa cells. We conclude from our results that the chemically-induced viscosity variations are an ultimate consequence of the alteration in the polymerization/depolymerization dynamics of the microtubules, rather than the actin filaments. This modifies the average size of the intracellular components, changing the effective viscosity that drags the movement of the nanorocker. A more detailed study could be done with a range of particle's sizes in order to assess the viscosity changes at different scales.

Our work shows the use of non-spherical upconverting nanoparticles as intracellular rheometric sensors to monitor changes in the intracellular viscosity produced by chemotherapeutic treatments. This will help with the development of new chemotherapies and single-cell studies.

\section{Experimental Section}

Experimental setup: The $980 \mathrm{~nm}$ laser radiation generated by a fiber-coupled laser diode (Lumics $\mathrm{GmbH}$ ) was collimated and expanded to slightly exceed the back aperture of the microscope objective (Optika, LWDPLAN, $60 \times, \mathrm{NA}=0.7$ ) that was used to create a diffraction-limited beam spot. We used the same objective to collect the luminescence of the excited UCNP and to image the sample plane using a CMOS camera (Thorlabs, DCC1645C). The spectral distribution of the nanoparticle's luminescence was analyzed using a CCD 


\section{WILEY-VCH}

camera (Horiba, Synapse) coupled to a monochromator (Horiba, iH320). We used optical filters (Thorlabs, FESH950) to block the laser light from reaching both cameras.

We analyze the cells directly in the same petri dish in which they were grown, incubated with the UCNPs and treated. They were always kept immersed in the culture medium. We kept the cells in a warm environment and translated individual dishes to the optical setup for the viscosity measurements. We continuously monitored the cell state during the experiments and we discarded samples if any sign of deterioration was observed.

Cell preparation for chemotherapy studies: We cultured HeLa cancer cells (ATCC) to 50 $60 \%$ confluence at $37{ }^{\circ} \mathrm{C}$ in $10 \%$ Dulbecco's Modified Eagle's Medium supplemented with $10 \%$ bovine calf serum, 50 units $/ \mathrm{mL}$ penicillin and $50 \mathrm{~g} / \mathrm{mL}$ streptomycin (Sigma). To introduce the luminescent probes inside the cells, we incubate them for $2 \mathrm{~h}$ with a diluted colloidal solution of the nanorockers in PBS. Then, we washed the cultures several times to eliminate the particles not internalized by the cells. After that, we subjected the cells to different chemical treatments. To alter the microtubules of the cytoskeleton and, thus modify the intracellular viscosity, we treated the cells for different periods of time $(0.5,1,2,3$, and 5 h) with $0.2 \mu \mathrm{M}$ paclitaxel (Taxol) to promote polymerization of tubulin into microtubules or with 0.1 , and $1 \mu \mathrm{M}$ colchicine for $2 \mathrm{~h}$, to collapse cellular microtubules. Colchicine and paclitaxel were purchased from Sigma Chemical (St. Louis, MO). After all drug treatments, we washed the cultures several times to remove the chemicals from the culture medium. Rheometric technique: The viscosity of the medium is related to the rocking movement of the particle through the mean square angular displacement (MSAD) (see Equation 1). We obtain this parameter from the evolution of the angular displacement of the particle $(\theta)$ with time (i.e. $M S A D(t)=\sum_{t=0}^{t_{i}}\left(\theta_{i}-\bar{\theta}\right)^{2}$, where $\theta_{i}$ is the orientation of the particle at time $t_{i}$ and $\bar{\theta}$ is the mean value of the angular displacement). We calculated the angular displacement at a given time from the emission spectrum using Equation 2. To do so, we analyze the polarized 


\section{WILEY-VCH}

emission of the particle to determine its orientation. We obtain the maximum value of the intensity ratio between the peaks centered at $654 \mathrm{~nm}$ and $661 \mathrm{~nm}$ (corresponding to the vertical orientation) from the set of spectra (see Figure $2 a$ and $b$ ). The minimum intensity ratio is set to a reference value that we obtain as the mean value of the intensity ratio of a set of UCNPs stuck on a surface in the horizontal orientation. We tested possible variations between the actual minimum ratio and the reference value and we saw that results deviated less than the experimental uncertainty. We used this maximum and minimum values in Equation 2, together with the intensity ratio computed at each time instant, to obtain the angular displacement $\theta_{i}$. Then, we obtain the evolution of the MSAD and fitted it to a linear function to obtain a value of the viscosity. The values we used for the volume and Perrin's friction coefficient were calculated from the mean particle's size obtained from SEM images. We used the luminescence emission of the UCNPs to locate them within the cells. We only used one particle per cell. Once a UCNP was found in the same location as a cell, we check its internalization. To do so, we changed the position of the excitation focus to ensure that the maximum detected intensity corresponded to a plane within the volume of the cell. From this we concluded that the particle was internalized rather than on the upper membrane of the cell. Cell preparation for immunodetection assay: We grew cells on round coverslips placed into 24-well plates. For tubulin detection (microtubule visualization), we fixed the cells in cold methanol $\left(-20^{\circ} \mathrm{C}\right)$ for $7 \mathrm{~min}$. After washed in PBS, we incubated the cells with alpha-tubulin (Sigma) primary antibody for $1 \mathrm{~h}$ at $4{ }^{\circ} \mathrm{C}$, in $0.1 \%$ PBS/BSA. Then we washed the cells twice with PBS and incubate them with Alexa-488 (Invitrogen) secondary antibody (1:500 in 0.1\% PBS/BSA), also for $1 \mathrm{~h}$ at $4{ }^{\circ} \mathrm{C}$. Finally, we washed the cells again. For F-actin detection (actin filaments visualization), we fix, stained and permeabilized the cells in a single step by the addition of a $2 X$ cold solution containing 5 units/ml Alexa 546labeled phalloidin (Molecular Probes), $0.5 \mathrm{mg} / \mathrm{ml}$ L- $\alpha$-lysophosphatidyl-choline, and $3.5 \%$ 
formaldehyde in PBS. After 20 min at $4{ }^{\circ} \mathrm{C}$, we washed the cells twice in cold PBS. Finally, all samples were mounted in Prolong-GOLD (Molecular Probes).

\section{Supporting Information}

Supporting Information is available from the Wiley Online Library or from the author.

\section{Acknowledgements}

This work was partially supported by the Ministerio de Economía y Competitividad de España (MAT2016-75362-C3-1-R and MAT2017-83111R), by the Instituto de Salud Carlos III (PI16/ 00812), by the Comunidad Autónoma de Madrid (B2017/BMD- 3867RENIMCM), and co-financed by the European Structural funds and also by COST action CM1403. R.D-B and R.P.P acknowledge funding from Comunidad de Madrid predoctorate contracts and from the Spanish national project FIS2017-86007-C3-1-P. We also gratefully acknowledge the support of the NVIDIA Corporation with the hardware donations used for this research. Research data supporting this publication can be accessed at https://doi.org/10.17630/c556bd9b-f995-4c74-a645-606ef2eb5621

Received: ((will be filled in by the editorial staff)) Revised: ((will be filled in by the editorial staff)) Published online: ((will be filled in by the editorial staff))

\section{References}

1. a) Wirtz, D., Particle-Tracking Microrheology of Living Cells: Principles and Applications. In Annual Review of Biophysics, 2009; Vol. 38, pp 301-326; b) Liu, T.; Liu, X.; Spring, D. R.; Qian, X.; Cui, J.; Xu, Z., Scientific Reports 2014, 4, 5418; c) Suresh, S.; Spatz, J.; Mills, J. P.; Micoulet, A.; Dao, M.; Lim, C. T.; Beil, M.; Seufferlein, T., Acta Biomaterialia 2005, 1 (1), 15-30.

2. $\quad$ a) Yun, X.; Tang, M.; Yang, Z.; Wilksch, J. J.; Xiu, P.; Gao, H.; Zhang, F.; Wang, H., RSC Advances 2017, 7 (69), 43764-43771; b) Tsai, M. A.; Waugh, R. E.; Keng, P. C., Biophys. J. 1998, 74 (6), 3282-3291; c) Ying-chun, C.; Chien-ming, W., Japanese Journal of Applied Physics 2012, 51 (12R), 127001.

3. Huber, F.; Boire, A.; López, M. P.; Koenderink, G. H., Curr. Opin. Cell Biol. 2015, $32,39-47$.

4. Silvis, N. G., Dermatol. Clin. 2001, 19 (1), 105-118. 
5. $\quad$ a) Manasa, K. L., Int. J. Pharm.l Sci. Res. 2015, 6 (9), 3713-3725; b) Cassimeris, L. U.; Walker, R. A.; Pryer, N. K.; Salmon, E. D., BioEssays 1987, 7 (4), 149-154; c) Avila, J., The FASEB Journal 1990, 4 (15), 3284-3290.

6. Penna, L. S.; Henriques, J. A. P.; Bonatto, D., Pharmacol. Ther. 2017, 173, 67-82.

7. Sahil, S.; Manish Kumar, G.; Ajit Kumar, S.; Preet Mohinder Singh, B., Anti-Cancer Agents Med. Chem. 2017, 17 (2), 230-240.

8. Mahindroo, N.; Liou, J.-P.; Chang, J.-Y.; Hsieh, H.-P., Expert Opin. Ther. Pat. 2006, $16(5), 647-691$.

9. $\quad$ a) Jordan, M. A.; Thrower, D.; Wilson, L., J. Cell Sci. 1992, 102 (3), 401-416; b)

Boyd, A. E.; Bolton, W. E.; Brinkley, B. R., The Journal of Cell Biology 1982, 92 (2), 425 434; c) White, J. G., The American Journal of Pathology 1968, 53 (2), 281-291.

10. Singh, H.; Kumar, M.; Nepali, K.; Gupta, M. K.; Saxena, A. K.; Sharma, S.; Bedi, P. M. S., Eur. J. Med. Chem. 2016, 116, 102-115.

11. Chakraborty, S.; Rahman, T., Ecancermedicalscience 2012, 6, ed16-ed16.

12. a) Puchkov, E. O., Biochemistry (Moscow) Supplement Series A: Membrane and Cell Biology 2013, 7 (4), 270-279; b) Ayala, Y. A.; Pontes, B.; Ether, D. S.; Pires, L. B.; Araujo, G. R.; Frases, S.; Romão, L. F.; Farina, M.; Moura-Neto, V.; Viana, N. B.; Nussenzveig, H. M., BMC Biophysics 2016, 9 (1), 5; c) Rodríguez-Sevilla, P.; Labrador-Páez, L.; HaroGonzález, P., Optical Materials 2018, 84, 514-523.

13. a) Berret, J. F., Nat. Commun. 2016, 7, 10134; b) Sleep, J.; Wilson, D.; Simmons, R.; Gratzer, W., Biophys. J. 1999, 77 (6), 3085-3095; c) Yousafzai, M. S.; Ndoye, F.; Coceano, G.; Niemela, J.; Bonin, S.; Scoles, G.; Cojoc, D., Optics and Lasers in Engineering 2016, 76, 27-33.

14. a) Chen, Y.-Q.; Kuo, C.-Y.; Wei, M.-T.; Wu, K.; Su, P.-T.; Huang, C.-S.; Chiou, A., J. Biomed. Opt. 2013, 19 (1), 011008-011008; b) Chevry, L.; Colin, R.; Abou, B.; Berret, J.F., Biomaterials 2013, 34 (27), 6299-6305; c) Lee, J. S. H.; Panorchan, P.; Hale, C. M.; Khatau, S. B.; Kole, T. P.; Tseng, Y.; Wirtz, D., J. Cell Sci. 2006, 119 (9), 1760-1768; d) Reuel, N. F.; Dupont, A.; Thouvenin, O.; Lamb, D. C.; Strano, M. S., ACS Nano 2012, 6 (6), 5420-5428; e) Tseng, Y.; Lee, J. S. H.; Kole, T. P.; Jiang, I.; Wirtz, D., J. Cell Sci. 2004, 117 (10), 2159-2167; f) Yamada, S.; Wirtz, D.; Kuo, S. C., Biophys. J. 2000, 78 (4), 1736-1747. 15. a) Kuimova, M. K.; Yahioglu, G.; Levitt, J. A.; Suhling, K., J. Am. Chem. Soc. 2008, 130 (21), 6672-6673; b) Kuimova, M. K.; Botchway, S. W.; Parker, A. W.; Balaz, M.;

Collins, H. A.; Anderson, H. L.; Suhling, K.; Ogilby, P. R., Nat Chem 2009, 1 (1), 69-73; c) Fushimi, K.; Verkman, A. S., J. Cell Biol. 1991, 112 (4), 719-725.

16. a) Nadort, A.; Zhao, J.; Goldys, E. M., Nanoscale 2016, 8 (27), 13099-13130; b) Zhou, B.; Shi, B.; Jin, D.; Liu, X., Nat. Nanotechnol. 2015, 10 (11), 924-936; c) Liang, L.; Qin, X.; Zheng, K.; Liu, X., Acc. Chem. Res. 2019, 52 (1), 228-236.

17. Rodríguez-Sevilla, P.; Zhang, Y.; de Sousa, N.; Marqués, M. I.; Sanz-Rodríguez, F.; Jaque, D.; Liu, X.; Haro-González, P., Nano Lett. 2016, 16 (12), 8005-8014.

18. Kalwarczyk, T.; Ziębacz, N.; Bielejewska, A.; Zaboklicka, E.; Koynov, K.;

Szymański, J. d.; Wilk, A.; Patkowski, A.; Gapiński, J.; Butt, H.-J. r.; Hołyst, R., Nano Lett. 2011, 11 (5), 2157-2163.

19. Erb, R. M.; Segmehl, J.; Charilaou, M.; Loffler, J. F.; Studart, A. R., Soft Matter 2012, 8 (29), 7604-7609.

20. van den Broek, B.; Ashcroft, B.; Oosterkamp, T. H.; van Noort, J., Nano Lett. 2013, 13 (3), 980-986.

21. Rodríguez-Sevilla, P.; Zhang, Y.; Sousa, N. d.; Marqués, M. I.; Sanz-Rodríguez, F.; Jaque, D.; Liu, X.; Haro-González, P. In Microrheometric upconversion-based techniques for intracellular viscosity measurements, SPIE Nanoscience + Engineering, SPIE: 2017; p 13. 


\section{WILEY-VCH}

22. Jordan, M. A.; Toso, R. J.; Thrower, D.; Wilson, L., Proc. Natl. Acad. Sci. U. S. A. 1993, 90 (20), 9552-9556.

23. Wang, X.; Tanaka, M.; Krstin, S.; Peixoto, H. S.; Wink, M., Molecules (Basel, Switzerland) 2016, 21 (7), 906.

24. Hohmann, T.; Dehghani, F., Cells 2019, 8 (4), 362.

25. Mozziconacci, J.; Sandblad, L.; Wachsmuth, M.; Brunner, D.; Karsenti, E., PLoS One 2008, 3 (11), e3821.

26. Solomon, M. J.; Spicer, P. T., Soft Matter 2010, 6 (7), 1391-1400.

27. a) Edmond, K. V.; Elsesser, M. T.; Hunter, G. L.; Pine, D. J.; Weeks, E. R.,

Proceedings of the National Academy of Sciences 2012, 109 (44), 17891-17896; b) Alcázar-

Cano, N.; Delgado-Buscalioni, R., Soft Matter 2018, 14 (48), 9937-9949.

28. a) Vázquez-Quesada, A.; Usabiaga, F. B.; Delgado-Buscalioni, R., The Journal of Chemical Physics 2014, 141 (20), 204102; b) Balboa Usabiaga, F.; Delgado-Buscalioni, R.; Griffith, B. E.; Donev, A., Computer Methods in Applied Mechanics and Engineering 2014, 269, 139-172. 


\section{WILEY-VCH}

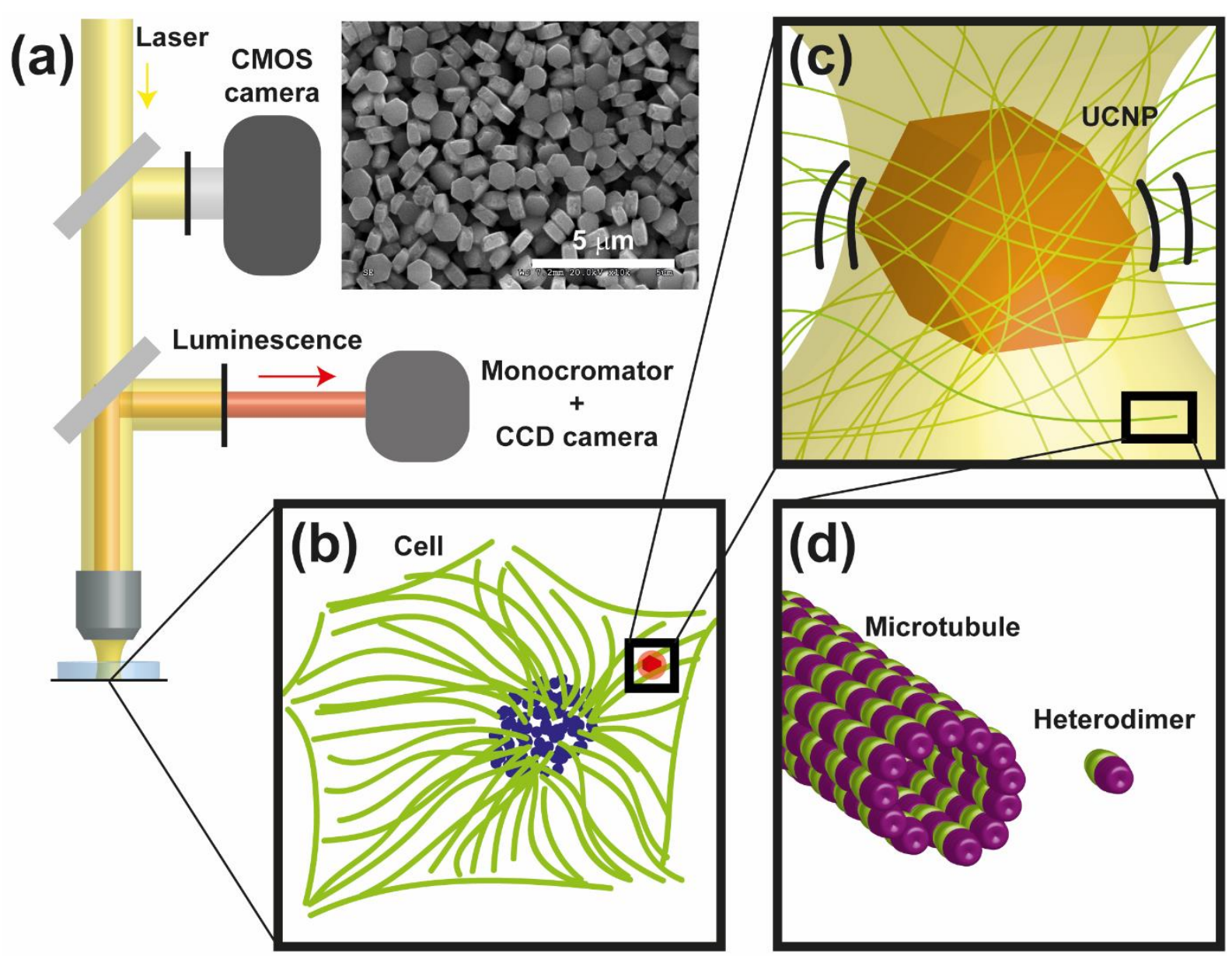

Figure 1. (a) Schematic representation of the experimental setup used to measure the intracellular viscosity composed by the excitation beam, a CMOS camera to visualize the sample, and a monochromator coupled to a CCD camera to measure the luminescence spectrum of the internalized upconverting nanoparticles (UCNPs). A typical SEM image of the UCNPs is included. (b) HeLa cells are incubated with the UCNPs and subjected to the different drug treatments. Once inside the cell, (c) the UCNP is surrounded by microtubules that affect its Brownian motion. The microtubules are (d) hollow tubes formed by heterodimers that dynamically assembly and disassembly. 

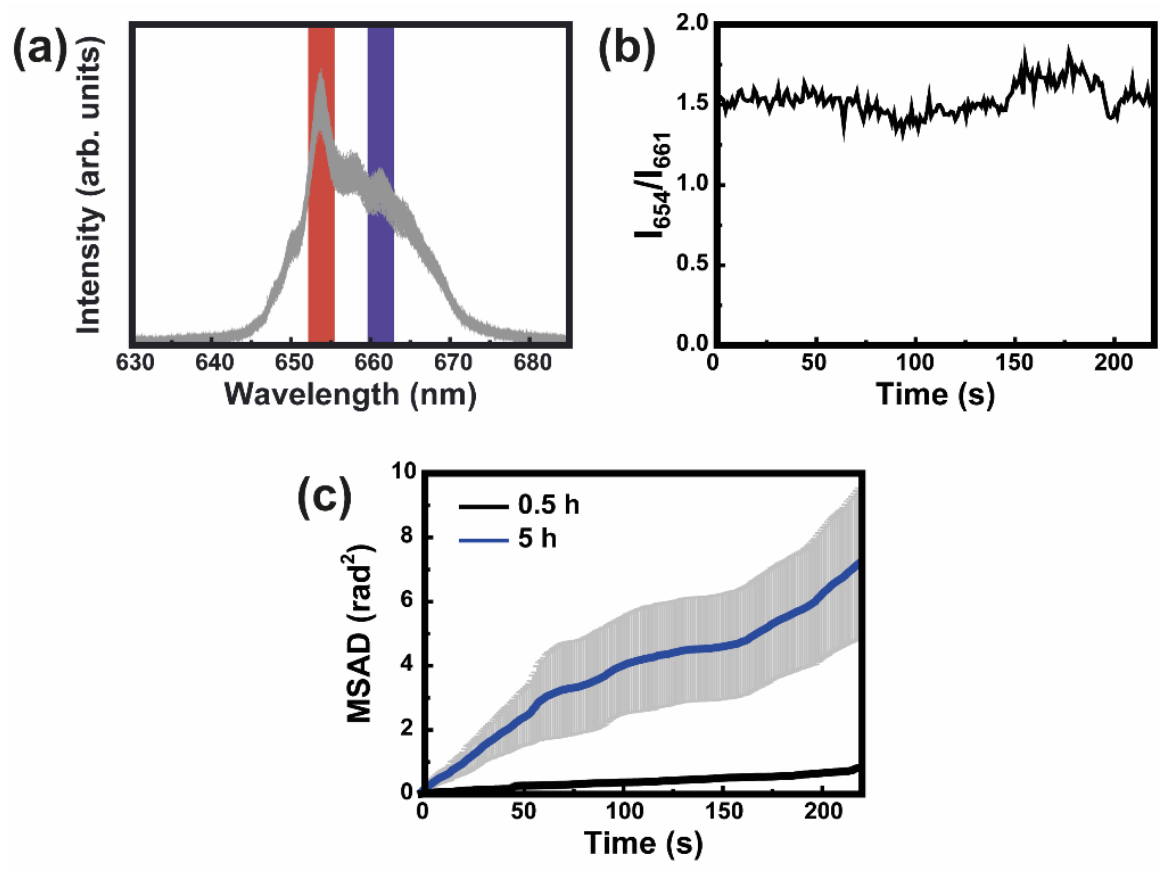

Figure 2. For the determination of the intracellular viscosity, we measure (a) consecutive luminescent spectra from a single nanorocker located inside a HeLa cell. We then use the highlighted peaks to determine the (b) intensity ratio $\left(\mathrm{I}_{654} / \mathrm{I}_{661}\right)$ that gives the angular position of the particle through Equation 2. We finally use this angular position as a function of time to calculate the (c) evolution of the mean square angular displacement (MSAD) which we used to determine the intracellular viscosity using Equation 1. Black and blue curves correspond to the mean value of the MSAD of particles internalized in Taxol-treated cells incubated for 0.5 and $5 \mathrm{~h}$, respectively. Gray bars represent the statistical error of different measurements. 
WILEY-VCH
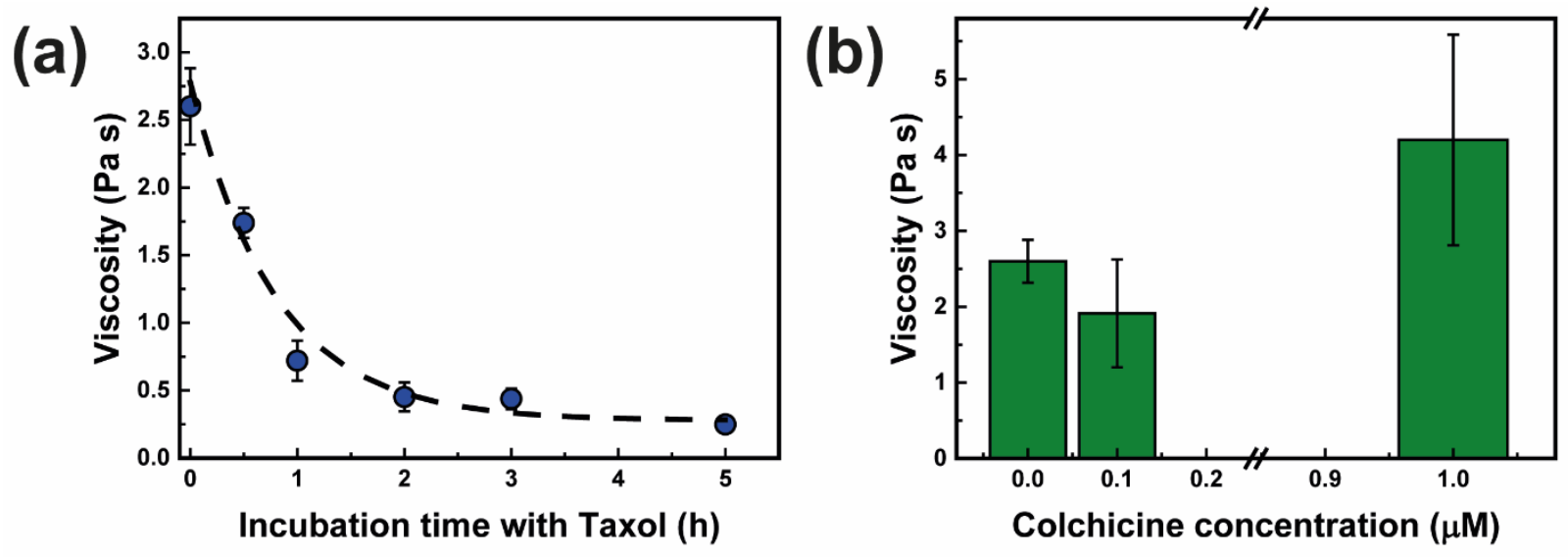

Figure 3. (a) Decrease in the intracellular viscosity as the incubation time with Taxol (at a concentration of $0.2 \mu \mathrm{M}$ ) increases. Blue data represents the experimental results, while the black dashed line is the best exponential decay fit. (b) Intracellular viscosity for cells incubated for $2 \mathrm{~h}$ with two different colchicine concentrations. Only the higher concentration of $1.0 \mu \mathrm{M}$ produces an increase in the viscosity. Data correspond to the mean obtained value and error bars represent the standard deviation of all measurements performed for each treatment conditions. Results for untreated cells are also shown. 


\section{WILEY-VCH}

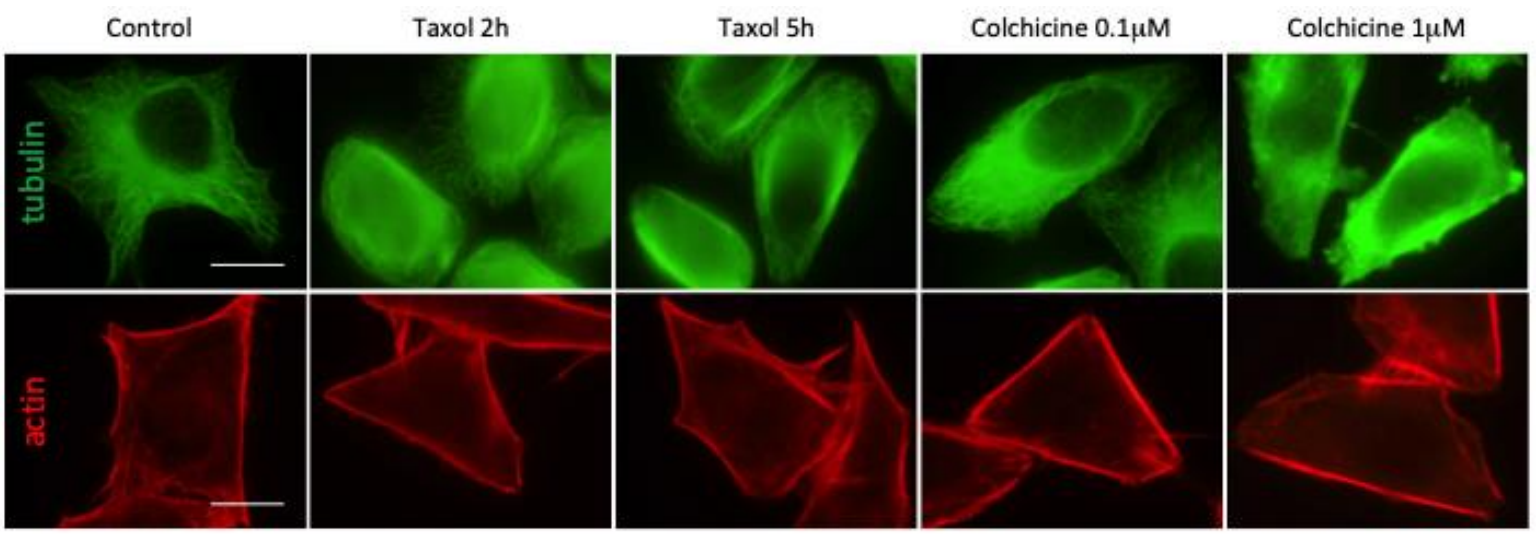

Figure 4. Fluorescence images of two components of the cytoskeleton of untreated and treated HeLa cells. Tubulin is marked in green, while actin is colored in red. Control cells were not subjected to any chemical treatment. Cells incubated with Taxol at a concentration of $0.2 \mu \mathrm{M}$ for 2 and $5 \mathrm{~h}$ (second a third columns, respectively) present microtubules forming bundles due to the stabilization produced by this drug. On the other hand, the microtubule network of cells treated with $1 \mu \mathrm{M}$ of colchicine (fifth column) is partially destroyed. This effect cannot be seen in cell incubated at 0.1 colchicine concentration (fourth column). Actin filaments do not present alterations due to the drug treatments. Only the microtubule network (formed by tubulin) is directly affected during the drug treatments. Scale bar is $10 \mu \mathrm{m}$. 


\section{WILEY-VCH}
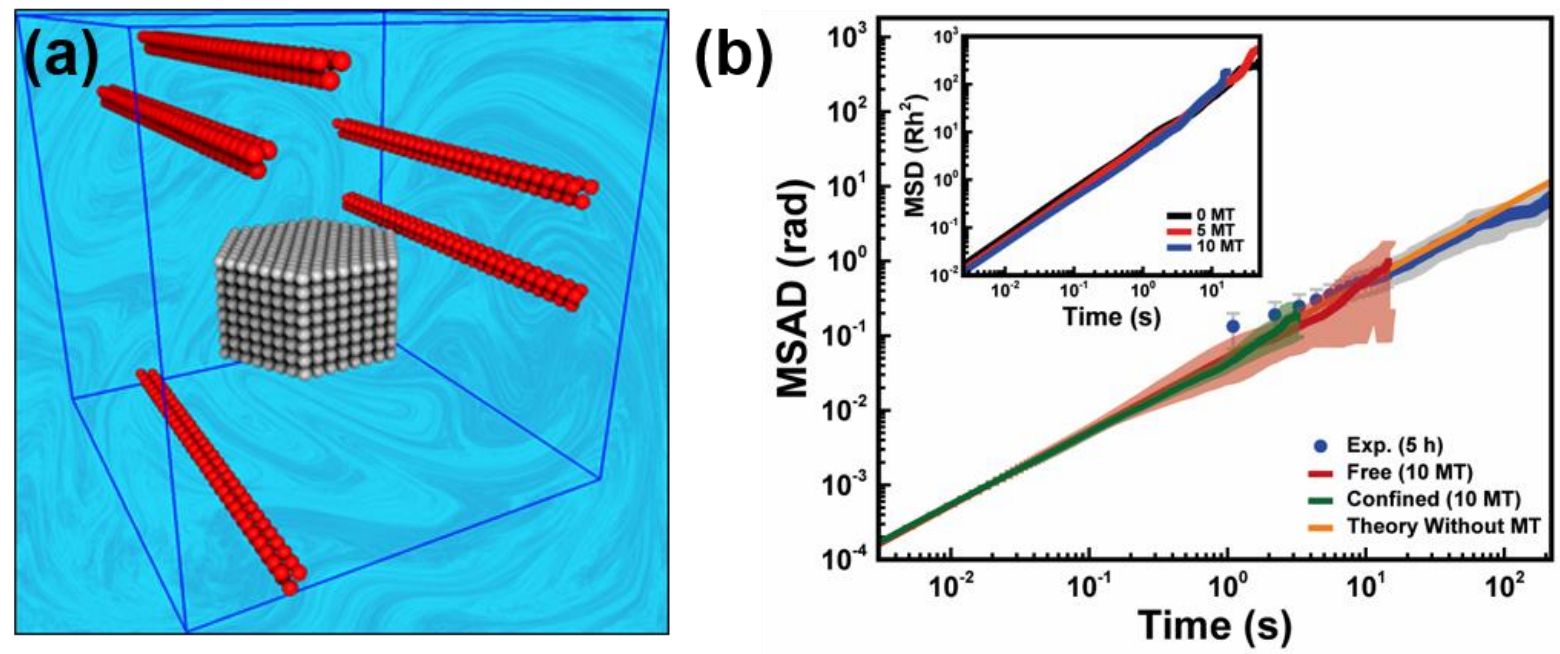

Figure 5. (a) Representation of the different elements of the computational model used to study the effect of microtubules in the dynamics of the nanorocker. (b) Computed and experimentally measured mean squared angular displacement (MSAD) over time.

Comparison is made between simulations of free (red line) and laser-illuminated (confined, green line) nanorocker, surrounded by 10 microtubules (MT), and the experimental results (Exp.) after $5 \mathrm{~h}$ incubation time with Taxol (blue data). The orange line represents the MSAD calculated from Equation 1 using the measured viscosity for an incubation time of $5 \mathrm{~h}$. The good agreement between orange and blue curves validates the approximation of the nanorocker to a disk. The inset shows the mean squared displacement (MSD) for free nanorockers in the presence of 0,5 and 10 microtubules. The agreement between the experimental and simulation results indicates that the movement of the particle is not affected by the presence of microtubules since their size is much bigger than the particle. 